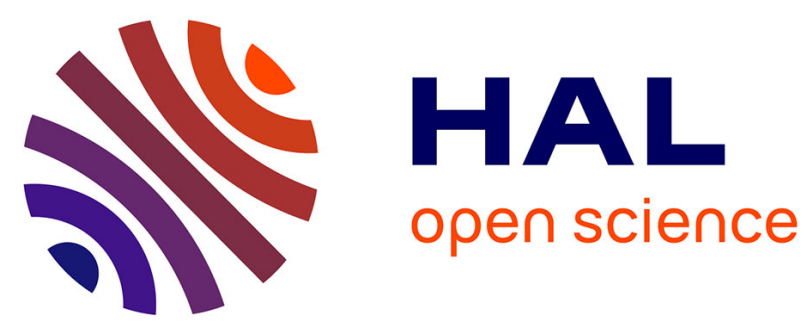

\title{
Grain growth: The key to understand solid-state dewetting of silver thin films
}

P Jacquet, Renaud Podor, J Ravaux, J Teisseire, I Gozhyk, J Jupille, R Lazzari

\section{- To cite this version:}

P Jacquet, Renaud Podor, J Ravaux, J Teisseire, I Gozhyk, et al.. Grain growth: The key to understand solid-state dewetting of silver thin films. Scripta Materialia, 2016, 115, pp.128-132. 10.1016/j.scriptamat.2016.01.005 . hal-01274691

\section{HAL Id: hal-01274691 \\ https://hal.science/hal-01274691}

Submitted on 16 Feb 2016

HAL is a multi-disciplinary open access archive for the deposit and dissemination of scientific research documents, whether they are published or not. The documents may come from teaching and research institutions in France or abroad, or from public or private research centers.
L'archive ouverte pluridisciplinaire HAL, est destinée au dépôt et à la diffusion de documents scientifiques de niveau recherche, publiés ou non, émanant des établissements d'enseignement et de recherche français ou étrangers, des laboratoires publics ou privés. 


\title{
Grain growth : the key to understand solid-state dewetting of silver thin films
}

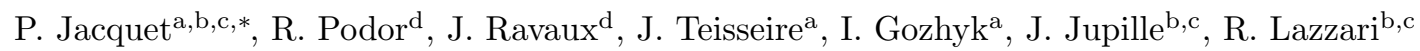 \\ ${ }^{a}$ Surface du Verre et Interfaces, UMR 125 CNRS/Saint-Gobain Recherche, 39 quai Lucien Lefranc, F-93303 Aubervilliers, France \\ ${ }^{b}$ CNRS, UMR 7588, Institut des NanoSciences de Paris, 4 place Jussieu, F-75252 Paris, France \\ ${ }^{c}$ Sorbonne Universités, UPMC Univ Paris 06, UMR 7588, Institut des NanoSciences de Paris, 4 place Jussieu, F-75252 Paris, France \\ ${ }^{d}$ Institut de Chimie Séparative de Marcoule, UMR 5257 CEA-CNRS-UM-ENSCM, Site de Marcoule, F-30207 Bagnols sur Cèze, France
}

\begin{abstract}
The dynamics of solid-state dewetting of polycrystalline silver thin films in oxygen atmosphere was investigated with in situ and real-time environmental Scanning Electron Microscopy at high temperature combined with Atomic Force Microscopy. Three steps were identified during dewetting : induction, hole propagation without specific rim and sintering. Moreover, it was observed that a very selective grain growth, promoted by surface diffusion, plays a key role all along the process.
\end{abstract}

Keywords: Solid-state dewetting, grain growth, surface diffusion, Scanning Electron Microscopy, thin films

Continuous polycrystalline metallic films are metastable when deposited on a substrate they do not wet [1]. Their spontaneous evolution with time or upon appropriate annealing results in very specific nanostructures or patterns with high potential of applications in optics [2] and optoelectronics [3]. This process is called dewetting, because the initial continuous layer breaks up into particles 4, as would a water film do on a hydrophobic surface. This transformation is governed by the decrease of the total interfacial energy of the system [4, 5]. Yet in the case of solid-state dewetting, it happens through diffusive mass transport along the surface, the interface or grain boundaries 4, 6, 7. Moreover, as solid crystalline surfaces exhibit inherent anisotropy, the knowledge of diffusion paths is crucial to understand the dewetting kinetics 8 and the resulting morphology of the dewetting layer [9].

So far, dewetting was commonly described to occur in two steps. First, during an induction or incubation period, hillocks appear although the substrate coverage by the film is apparently not modified [4. Hillock growth is often assumed to result from the compressive stress arising from differential thermal expansion upon annealing [4. In the second step, holes appear within the film and grow [4, 5, 10]. Hole expansion is due to curvature differences between the dewetting front and a propagating rim [1, 11]. Indeed, according to Fick's first law, the driving forces for diffusion are local fluctuations in chemical potential which is directly linked to surface curvature [11. Finally, the rim is transformed into isolated islands due to Rayleigh instabilities 11. However, the exact morphology of both dewetting front and propagating rim have been

* Corresponding author long-discussed. In the first capillary models, the rim was round shaped [11, 12] and the layer behind it was depleted 11]. Later, Jiran and Thompson [8, suggested that the front was as thick as the layer without any rim. Surface energy anisotropy was considered later on, but on a rim observed in dewetting on single-crystals [13, 14. Based on ex situ observations, they adapted capillary approach to single-crystals case by including solid diffusion.

More recently, the role of grains in dewetting of polycrystalline layers started to be discussed in the literature [10, 15, 16, 17. In this regard, new insight on the role of grains and dewetting in general was brought by in situ techniques, naturally allowing for more accurate kinetic data compared to interrupted annealing 18 Kosinova et al. 6, 9] studied hole propagation by in situ Atomic Force Microscopy (AFM). They observed grain growth at the edge of the hole and behind the first grain row. A new model was suggested, where hole propagation was due to successive growth and shrinkage of neighboring grains coupled by interface diffusion. This diffusion was found to depend on the surrounding atmosphere [6, 9]. Atiya et al. 16, 17. showed that the dewetting of platinum thin films supported on $\mathrm{SrTiO}_{3}(100)$ is clearly impacted by the orientation of the substrate. They observed that grains having specific crystallographic orientations play an important role in hole propagation morphology and kinetics. However, real-time imaging of the kinetics of dewetting is still lacking to really unravel the evolution and actual existence of a dewetting rim and the role of grains in the process. The present work is dealing with the dewetting morphology of a polycrystalline silver thin film as observed by in situ real-time Environmental Scanning Electron Microscopy (ESEM) and AFM. Amorphous silica was se- 
lected as a substrate to get rid of epitaxy issues and its poor surface energy anisotropy [19 makes silver a good candidate for capillary approach. Moreover, its sizable surface diffusion in oxygen [20] allows experiments to be run at moderate temperatures. Our results show that dewetting proceeds in three steps: induction, hole propagation and sintering, which are driven to a large extent by the extraordinary growth of specific grains.

All studied films of 20 or $40 \mathrm{~nm}( \pm 1 \mathrm{~nm})$ thicknesses were deposited at room temperature by pulsed current magnetron sputtering (power: $210 \mathrm{~W}$; deposition rate : $1.3 \mathrm{~nm} / \mathrm{s}$; Ar pressure : $8.10^{-3} \mathrm{mbar}$ ) on silicon wafers covered with their native oxide. Thicknesses were determined by AFM step measurement and the initial film roughnesses (measured by AFM) were in the range of $5.0 \mathrm{~nm}$ (Root Mean Square). SEM experiments were performed with a FEI Quanta 200 ESEM FEG apparatus in a $400 \mathrm{~Pa}$ oxygen atmosphere. A dedicated in situ heating stage allowed controlling very accurately the sample temperature $\left(25-400^{\circ} \mathrm{C}\right)$ through a thermocouple placed close to the sample 21. The heating $\operatorname{ramp}\left(50^{\circ} \mathrm{C} / \mathrm{s}\right)$ was fast enough to reach the setpoint temperature $\left( \pm 5^{\circ} \mathrm{C}\right)$ before the apparent onset of hole propagation. Two magnifications were used: $\times 3000$ to have statistical data for morphology and coverage analysis and $\times 20000$ to investigate the dewetting mechanism and grain growth at smaller scale. The fast video rate (20 images/min) allowed following the dynamics of the dewetting front. A careful image processing was performed with Sckikit-image library in Python. After a $(2,2)$ median filtering, the segmentation parameter was chosen for each image as the lowest minimum between the two highest maxima of the gray level histogram. To ensure that measured values were statistically satisfying, test were done on cropped images. The measured intensity values remained unchanged (less than $2 \%$ difference), excluding any size effect. A Bruker Dimension Icon AFM equipped with an heating stage was used in tapping mode to image topography during dewetting in air. The sample was annealed (below $150^{\circ} \mathrm{C}$ ) for an appropriate time (as indicated below) and cooled down to ambient temperature to image topography.

Continuous SEM videos at different temperatures were recorded, from the continuous thin film state to the final particle state (Fig. 1] see supplementary material online; video 1 at $\times 3000$ magnification and $390^{\circ} \mathrm{C}$ and video 2 at $\times 20000$ magnification and $330^{\circ} \mathrm{C}$ ). The real-time monitoring shows distinctly that dewetting proceeds in three steps: (i) a period of induction/incubation where the covered surface hardly evolves 4, (ii) a hole propagation with a complex evolution of the dewetting front i.e. the limit between covered and uncovered surface, and (iii) a longlasting late evolution of isolated worm-like particles which become more regular and agglomerated. Although thermodynamics predicts the formation of round shaped particles, they are not observed even after $30 \mathrm{~min}$ annealing (see
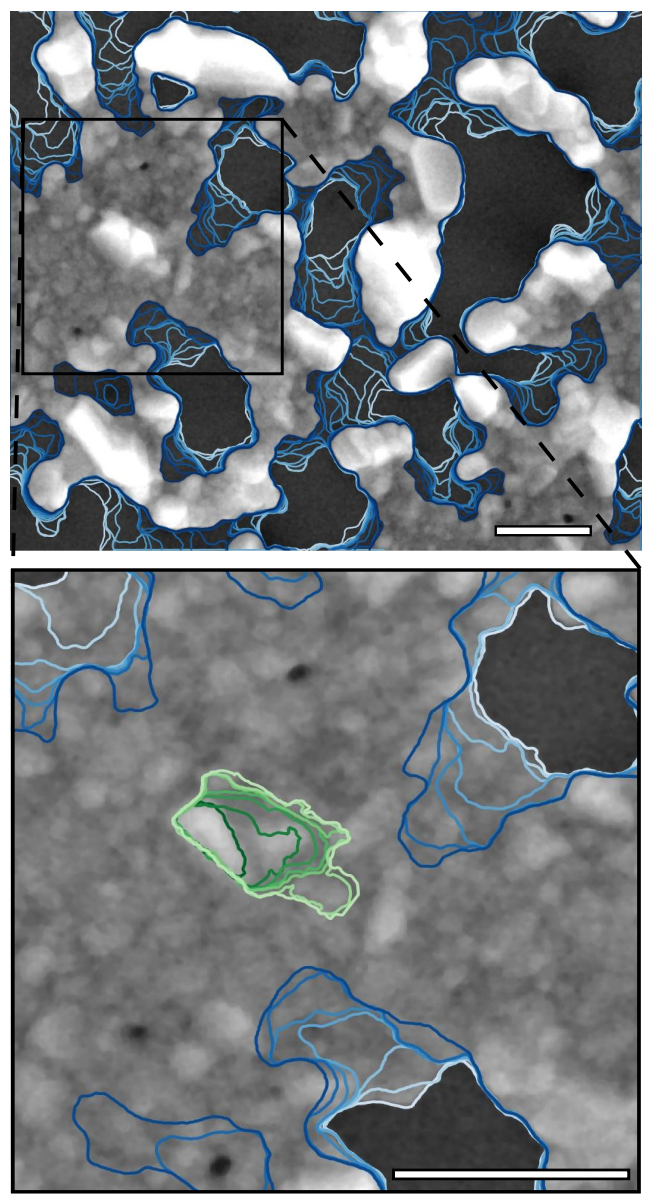

Figure 1: In situ SEM observation of solid-state dewetting on a $40 \mathrm{~nm}$ thick silver film on silica held at $330^{\circ} \mathrm{C}$ under $400 \mathrm{~Pa} \mathrm{O}_{2}$. Blue lines represent the time evolution of the front propagation, the lighter being the older. Green lines represent the grain boundary, the darker being the older. The scale bar is $1 \mu \mathrm{m}$.

video 2) suggesting that the dewetting is still not over. In the first studies [8], it was assumed that final particles were formed directly after the isolation of metal domains. Yet a more recent Tranmission Electron Microscopy work highlighted a film evolution after hole impingement [15, coherent with our observations. Extraordinary grain growth is another dominant feature of hole propagation in our present study on polycrystalline silver layers on amorphous substrate. It occurs close to the holes but also far away, as observed in iron/sapphire system [7]. For instance, in Fig. 1. the distance from the dewetting front (blue line) to a growing grain isolated on the continuous film (green line) is about $1 \mu \mathrm{m}$.

To rationalize these observations, images were analysed to extract the ratio of covered surface, the front length and the number of holes from video 1 (Fig. 2). Although the limit between induction and hole propagation is quite clear [4] i.e the onset of the variation of substrate coverage (marked by a full line in Fig. 2), the transition to sintering calls for a peculiar analysis. In Fig. 2, three events hap- 


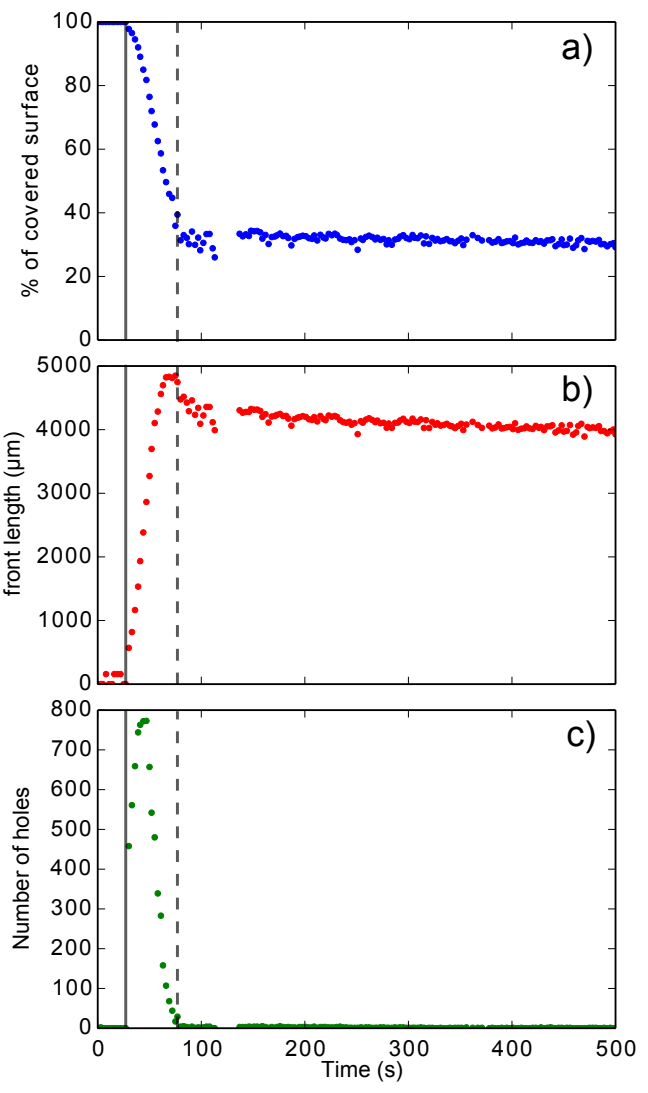

Figure 2: a) Covered surface, b) front length and c) number of holes versus time during dewetting of a silver layer at $390^{\circ} \mathrm{C}$ under $400 \mathrm{~Pa}$ $\mathrm{O}_{2}$. Data obtained from image analysis of video 1 in supplementary materials.

pen simultaneously (marked by a dashed line in Fig. 2 ) (i) the abrupt change of substrate uncovering rate, (ii) the maximum in front length, (iii) the impingement of holes into a percolated or interconnected domain. This specific time represents the limit when the entire initial layer has been modified by dewetting and when only agglomerated and isolated particles remain. When holes propagate, the front length increases, but on the contrary, it decreases when agglomerated particles sinter. In this state, besides slow Ostwald sintering, intra-particle mass transport is required to change the substrate coverage. This explains the variation of the uncovering rate, even with the same diffusion mechanisms. For these reasons, we suggest to define this time point as the global limit between hole propagation and sintering.

As already highlighted, all along the three-step process of dewetting on those polycrystalline layers, grain growth plays a key role. On video 2 of supplementary information, the image sequence begins shortly before the hole propagation step, at the end of induction time. At this point, grown grains are already observed (see Fig. 3 a). Since SEM electron contrast did not allow investigating their formation, the topography and roughness evolution of a $40 \mathrm{~nm}$ thick film was monitored during induction

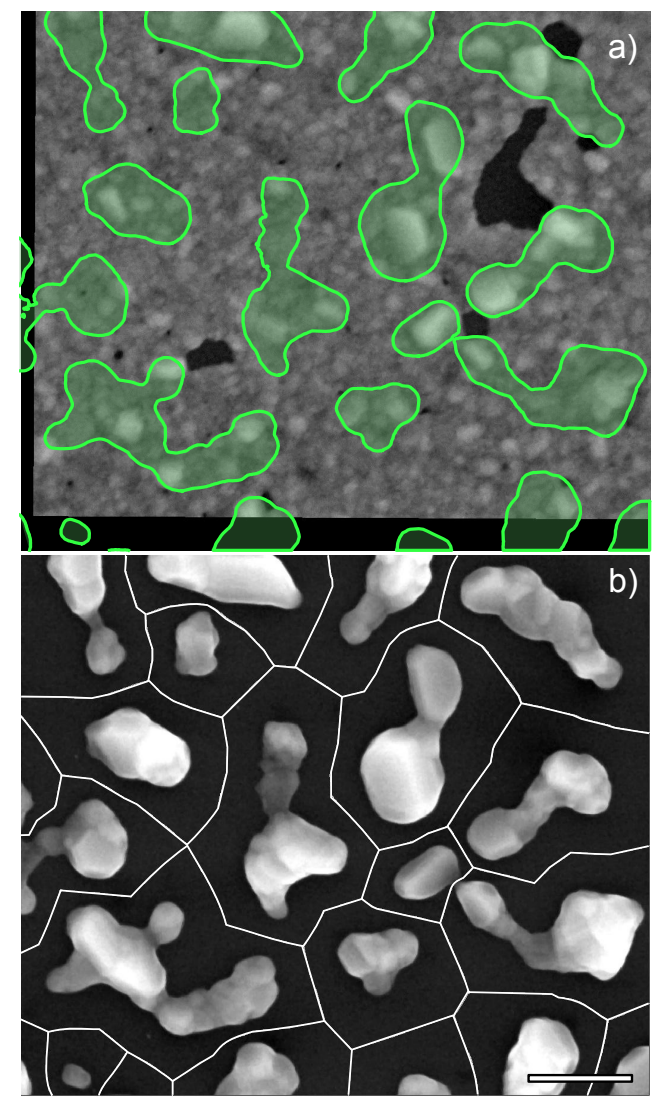

Figure 3: Comparison of two in situ SEM pictures : a) at the beginning of hole propagation. The shape of particles present in b) are superposed in green, b) at the beginning of sintering. Zones delimited with white lines represent the ensemble of points closer to the particle inside than any other one, the so-called "zone of influence". Scale bar is $1 \mu \mathrm{m}$.

by AFM. The results are presented in Fig. 4, at different times a) as deposited, b) after $5 \mathrm{~min}$ of annealing at $100^{\circ} \mathrm{C}$, c) after $5 \mathrm{~min}$ of annealing at $150^{\circ} \mathrm{C}$. The mean size of grains increases at each annealing treatment, as well as the roughness of the film: $3.9,4.7,7.2 \mathrm{~nm}$ respectively. Between each picture, some grains remained stable, other grains grew; some will go on growing giving birth to hillocks. At the latest stage of induction, although the determination may be limited by tip effects, it is possible to find holes with a depth of $20 \mathrm{~nm}$ equal to the layer thickness which could lead to hole formation, either by thermal grooving [10, 12, 22] or by shrinkage of smaller grains [23]. Unfortunately, the acquisition dynamics did not allow direct observation of hole birth. It is important to notice that even if the surface covered by the film is stable, induction is far from a static regime. The surface roughness of the film changes drastically and grain shape evolution may be seen as precursory of the next stage. The variety of observed behaviours could result from the absence of any epitaxy on amorphous silica.

At the end of induction time, the layer includes hillocks which appears as large grains, probably silver crystallites, 

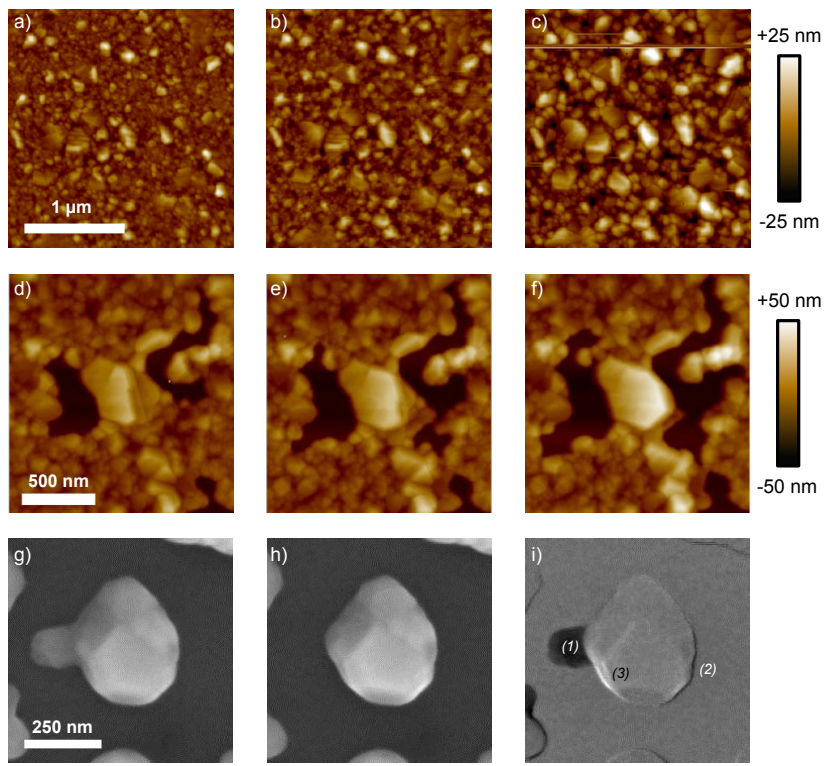

Figure 4: AFM images during induction time: a) as deposited (40 nm layer), b) after $10 \mathrm{~min}$ of annealing at $100^{\circ} \mathrm{C}$, c) after $5 \mathrm{~min}$ of annealing at $150^{\circ} \mathrm{C}$. Same image during hole propagation in a $20 \mathrm{~nm}$ silver layer annealed at $150^{\circ} \mathrm{C}$ : d) $6 \mathrm{~min}$, e) $9 \mathrm{~min}$, f) $15 \mathrm{~min}$. SEM images of a $40 \mathrm{~nm}$ silver layer annealed in $400 \mathrm{~Pa} \mathrm{O}_{2}$ at $330^{\circ} \mathrm{C}$ : g) reference picture, h) 5 min later, i) relative difference of the two images.

and holes (see Fig. 3 a). The observed morphology is very similar to the one described by Kovalenko et al. during iron on sapphire dewetting [7]. Holes propagate at the same time as hillocks continue growing (see video 2 ). In the present case, dewetting involve neither the propagation of a rim, nor a succession of growth and shrinkage of touching grains [6, 9]. Dewetting results from the propagation of holes, disappearance of small entities and abnormal growth of a limited number of grains. In other words, as already suggested in Ref. [7, a growing grain accumulates material from the initial layer contained in a "zone of influence" which can extend up to $1 \mu \mathrm{m}$ (see Fig. $3 \mathrm{~b}$ ). Moreover, the real-time in situ imaging allows to suggest (thanks to the big number of acquired images) that dewetting front does not propagate along grain boundaries as it was observed on $\mathrm{Au} / \mathrm{SiO}_{x}$ 15, but whole grains are shrinking instead (see front propagation in blue lines on Fig,1 or video 2).

Complementary AFM topography experiments were performed to better understand this issue. Similar morphologies were obtained by AFM upon dewetting in air or in low oxygen pressure in ESEM. Moreover, the mass conservation as observed through AFM profiles ruled out any sizeable effect of evaporation on the observed phenomena. During hole propagation on a $20 \mathrm{~nm}$ silver layer annealed at $150^{\circ} \mathrm{C}$ for 6,9 and $15 \mathrm{~min}$ (see Fig. 4 , d-f), the grain in the middle of the image is growing in a specific direction corresponding to a given facet (see AFM profiles in supplementary materials). The presence of grain boundary seems unlikely, and only a part of the grain is growing. Surface diffusion is governing grain growth, and thus could be a possible transport path, instead interface diffusion and grain boundary sliding as observed in other systems [6, 9], or grain boundary diffusion [7]. As shown in Fig. $3 \mathrm{a}$, the superposition of the shape of the particles just before the beginning of sintering, with the image at the beginning of the hole propagation (Fig $3 \mathrm{p}$ ) clearly highlights the pre-existence of preferential grains at the end of the induction. Clustering of grains that are to grow into bigger particles are easily identified in this way, which means that very specific grain, growing during induction, will grow further during hole propagation.

During the last sintering step, particles made of several grains tend to transform into single grains. Fig. $4 \mathrm{~g}, \mathrm{~h}, \mathrm{i}$ shows the sintering of a particle, initially formed by two grains (g), to a more regular shape (h). As images were taken in exactly the same conditions, the difference between the two pictures reveals qualitatively topology modifications (image difference Fig. 4 $\mathrm{fi}$ ). It demonstrates that silver has moved from the smaller grain (in dark, marked 1) and allowed the particle growth on specific facets (in light, marked 3). A slight displacement of the particle center of mass was also observed (marked 2), as it is commonly observed during sintering process.

These real-time SEM measurements shed light on a new description of the dewetting phenomenon in a polycrystalline layer. Dewetting proceeds in three steps: induction, hole propagation and sintering. Each step is deeply governed by grain growth. During induction, specific grains are selected and will accumulate the dewetting silver during the hole propagation step, even far away from the holes; no dewetting rim is observed. Then, a sintering step is observed, during which particles will slowly reach a shape closer to equilibrium. This uncommon dewetting scenario, which implies extraordinary grain growth, was already observed for iron but on a different substrate type (single crystal versus amorphous substrate) and in a different environment (reductive versus oxygen atmosphere) with other dominating diffusion path (interface versus surface) [7. The factors governing grain growth and grain selection are still to be understood to explain why these different systems exhibit so similar morphology evolution. Local fluctuations of grain sizes during deposition may drive the process but a simple size consideration is not sufficient to explain selectivity (Fig, 3 a). As the substrate herein is amorphous, crystallite epitaxy cannot explain this selection. This point is still under investigation and will probably be a key to understand and control dewetting.

[1] C. V. Thompson, Solid State Dewetting of Thin Films, Annu. Rev. Matter. 42 (2012) 399.

[2] H. Atwater, A. Polman, Plasmonics for improved photovoltaic devices., Nat. mater. 9 (3) (2010) 205-13.

[3] J. Ye, Fabrication of ordered arrays of micro- and nanoscale features with control over their shape and size via templated solid-state dewetting., Sci. Rep. 5 (November 2014) (2015) 9823. 
[4] A. Presland, G. Price, D. Trimm, Hillock formation by surface diffusion on thin silver films, Surf. Sci. 29 (2) (1972) $424-434$.

[5] S. K. Sharma, J. Spitz, HILlOCK FORMATION, HOLE GROWTH AND AGGLOMERATION IN THIN SILVER FILMS, Thin Solid Films 65 (1980) 339-350.

[6] A. Kosinova, L. Klinger, O. Kovalenko, E. Rabkin, The role of grain boundary sliding in solid-state dewetting of thin polycrystalline films, Scripta Mater. 82 (2014) 33-36.

[7] O. Kovalenko, J. Greer, E. Rabkin, Solid-state dewetting of thin iron films on sapphire substrates controlled by grain boundary diffusion, Acta Materialia 61 (2013) 3148-3156.

[8] E. Jiran, C. V. Thompson, Capillary Instabilities in Thin Films films, J. Electron. Mater. 19 (11) (1990) 1153.

[9] A. Kosinova, O. Kovalenko, L. Klinger, E. Rabkin, Mechanisms of solid-state dewetting of thin $\mathrm{Au}$ films in different annealing atmospheres, Acta Mater. 83 (2015) 91-101.

[10] R. Dannenberg, E. A. Stach, J. R. Groza, B. J. Dresser, Insitu TEM observations of abnormal grain growth, coarsening, and substrate de-wetting in nanocrystalline $\mathrm{Ag}$ thin films, Thin Solid Films 370 (2000) 54-62.

[11] R. Brandon, F. J. Bradshaw, The Mobility of the surface atoms of copper and silver evaporated deposits, Royal Aircraft Establishment, Tech. Rep. (66093).

[12] W. W. Mullins, Theory of Thermal Grooving, J. Appl. Phys. 28 (3) (1957) 333.

[13] L. Klinger, D. Amram, E. Rabkin, Kinetics of a retracting solid film edge: The case of high surface anisotropy, Scripta Mater. 64 (10) (2011) 962-965.

[14] R. V. Zucker, G. H. Kim, W. Craig Carter, C. V. Thompson, A model for solid-state dewetting of a fully-faceted thin film, C. R. Phys. 14 (7) (2013) 564-577.

[15] C. M. Müller, R. Spolenak, Microstructure evolution during dewetting in thin Au films, Acta Materialia 58 (2010) 60356045 .

[16] G. Atiya, D. Chatain, V. Mikhelashvili, G. Eisenstein, W. D. Kaplan, The role of abnormal grain growth on solid-state dewetting kinetics, Acta Mater. 81 (2014) 304-314.

[17] G. Atiya, V. Mikhelashvili, G. Eisenstein, W. D. Kaplan, Solidstate dewetting of $\mathrm{Pt}$ on (100) SrTiO3, J. Mater. Sci. 49 (11) (2014) 3863-3874.

[18] F. Niekiel, P. Schweizer, S. M. Kraschewski, B. Butz, E. Spiecker, The process of solid-state dewetting of Au thin films studied by in situ scanning transmission electron microscopy, Acta Mater. 90 (2015) 118-132.

[19] S. Stankic, R. Cortes-Huerto, N. Crivat, D. Demaille, J. Goniakowski, J. Jupille, Equilibrium shapes of supported silver clusters, Nanoscale 5 (2013) 2448-2453.

[20] G. E. Rhead, SELF-DIFFUSION AND FACETING ON SILVER, Acta Metall. 11 (1963) 1035.

[21] R. Podor, D. Pailhon, J. Ravaux, H.-P. Brau, Development of an Integrated Thermocouple for the Accurate Sample Temperature Measurement During High Temperature Environmental Scanning Electron Microscopy (HT-ESEM) Experiments., Microsc. Microanal. 21 (2) (2015) 307-312.

[22] W. W. Mullins, The effect of grooving on grain boundary motion, Acta Mater. 6 (1958) 414

[23] V. Derkach, A. Novick-Cohen, A. Vilenkin, E. Rabkin, Grain boundary migration and grooving in thin 3-D systems, Acta Materialia 65 (2014) 194-206. 